\title{
Sales Performance Management System
}

\author{
Dipesh Rane \\ Student B.E., \\ Department of Information \\ Technology, \\ MHSSCOE \\ Mumbai
}

\author{
Nabil Ahmed Mulla \\ Student B.E., \\ Department of Information \\ Technology, \\ MHSSCOE \\ Mumbai
}

\author{
Shabina Sayed \\ Assistant Professor, \\ Department of \\ Information Technology, \\ MHSSCOE \\ Mumbai
}

\author{
Daanish Sarguru \\ Student B.E., \\ Department of Information \\ Technology, \\ MHSSCOE \\ Mumbai
}

\begin{abstract}
An overwhelming amount of data is produced by an active sales team who store all this data in the form of handwritten notes, excel spreadsheets, text documents, or by memorizing it. The consequence of this is misplaced details, inconsistent follow up of meetings, and prioritising customers based on assumptions rather than facts.
\end{abstract}

Additionally, your customers may resolve their queries using different communication platforms, such as phone, email, and social media. Hence, if we don't have a standard platform for interacting with customers, this information exchange can be easily lost in the tremendous amount of information generated, resulting in poor feedback to your customers.

It will be stressful to derive intelligence from the vast amounts of collected data. This leads to difficulty in constructing and generating reports, which ultimately reduces productivity. A Manager's vision of what their teams are doing and where they stand begins to fade, resulting in failure to offer the right support when needed.

Sales Performance Management System for Mutual Funds aims to gather information from a variety of sources across your business and gives you unprecedented insights into how your customers feel and what they are saying about your organization. Hence, you can improve what you offer, spot problems early and identify gaps, and, ultimately, keep your customers happy with better service.

\section{Keywords}

Customer Relationship Management, CRM

\section{INTRODUCTION}

"Customer Relationship Management", often abbreviated as CRM, is a software that is used to store your contact details which includes their names, addresses, and phone numbers. Likewise, it enables the salesperson to maintain a record of all customer activity like website visits, phone calls, email, and also the tasks and events associated with them.

A CRM Software vigorously records and administers the customer information. If helps to connect your entire team from a device on which the corresponding CRM Software is maintained. The system intelligently captures customer emails regarding requests or complaints and allows you to generate tickets to resolve the associated matter. It streamlines and automates repetitive tasks so that the Salesperson concentrates mainly on the generating leads. One important feature of a CRM software is that you can extend it by adding new modules or integrating existing modules, and customize it as your business grows.

A CRM system assists your business to extend because it tracks the history of customer interactions. Tracking is important. Tracking related to modules such as calls made and emails sent, meetings held, presentations delivered, and even the next steps needed to close the deal or grow that customer account are carried out.

Customer relationship data should be automatically updated to run the business competently. Employees should access the data instantly and provides a full history of all communications, meetings, and documents shared.

A CRM system equips your business with a centralized storage to store to store all sales and marketing related data. The system is available on all platforms such as Desktop through Web Applications that can be run in a browser or Mobile through either Mobile Applications or Web Applications.

A CRM system can also be used by to get a tight grip over the prospective sales pipeline which makes forecasting easier, more detailed and correct.

\section{LITERATURE REVIEW 2.1 Need for the System}

Sales Performance Management System for Mutual Funds is a business solution that assist to preserve information about various activities scheduled for your existing customers and prospects. The system empowers the Relationship Manager to efficaciously manage and monitor their own performance, their team's performance, and their relationship's progress and past interactions. Progress at different levels of hierarchy can be coordinated by the system.

Sales Performance Management System for Mutual Funds expedites to create, modify, maintain, and associate the contacts with the Relationship Managers. Business relationship with contacts can be enhanced by tracking birthdays, anniversaries and other important events associated with them. Risk related to transfer or exit of relationship manager can be handled by the system. In such cases, all 
clients and business of that particular Relationship Manager will be assigned to another Relationship Manager.

Sales Performance Management System for Mutual Funds can generate reports, which the user can view, depending on the type of user and privileges the user has. The reports are generated using Business Analytics. Business Analytics consists of all programming modules to analyze data related to enterprise's customers and articulate it, so that better and quicker business decisions can be made.

Opportunity and the need to turn customer data into useful information has generally become evident as system is providing quicker and efficient interactivity with customers. [2]

\subsection{Existing Systems}

There are various existing CRM systems available, such as Microsoft Dynamics CRM, SugarCRM, SuiteCRM, Salesforce CRM, etc.
Table 1, given below, summarizes the differences between Salesforce CRM, Microsoft Dynamics CRM, SugarCRM, and SuiteCRM. It can be seen that although Salesforce and Dynamics CRM have the most number of features as required in a good CRM software, they possess little customizability and are costly, which may not be suitable for businesses that have differing requirements. SugarCRM and SuiteCRM are Open Source Software, which allows them to be customized by developers to suit their needs, but the former has disadvantages, as discussed above.

The Web Application for Sales Performance Management System will be built on top of SuiteCRM, which is the optimal choice considering the comparison shown in the table below. Although SuiteCRM lacks some functionalities that are offered by other CRM softwares, its advantage of being free and Open Source will be exploited to develop a CRM system to meet the requirements of a Mutual Funds organization. There is no licensing fee or other such costs involved, which reduces development and implementation costs. [6]

Table 1: Comparison of Existing CRM Systems ${ }^{[6]}$

\begin{tabular}{|c|c|c|c|c|}
\hline Feature & $\begin{array}{c}\text { Salesforce } \\
\text { Professional }\end{array}$ & $\begin{array}{l}\text { Microsoft Dynamics } \\
\text { CRM Professional }\end{array}$ & $\begin{array}{c}\text { SugarCRM } \\
\text { Professional }\end{array}$ & SuiteCRM \\
\hline Sales Automation & $\checkmark$ & $\checkmark$ & $\checkmark$ & $x$ \\
\hline Sales Collaboration & $\checkmark$ & $\checkmark$ & $\checkmark$ & $x$ \\
\hline Customer Interactions & $\checkmark$ & $\checkmark$ & $\checkmark$ & $\checkmark$ \\
\hline Contact Timelines & $\checkmark$ & $\checkmark$ & $\checkmark$ & $x$ \\
\hline Lead Generation & $\checkmark$ & $\checkmark$ & $\checkmark$ & $\checkmark$ \\
\hline Workflow & $x$ & $\checkmark$ & $\checkmark$ & $\checkmark$ \\
\hline Email Integration & $\checkmark$ & $\checkmark$ & $\checkmark$ & $\checkmark$ \\
\hline Email Marketing & $\checkmark$ & $\checkmark$ & $\checkmark$ & $\checkmark$ \\
\hline Inventory Management & $\checkmark$ & $\checkmark$ & $\checkmark$ & $x$ \\
\hline Employee Activity Tracking & $\checkmark$ & $\checkmark$ & $x$ & $\checkmark$ \\
\hline Group Calendar & $\checkmark$ & $\checkmark$ & $\checkmark$ & $\checkmark$ \\
\hline Performance Tracking & $\checkmark$ & $\checkmark$ & $\checkmark$ & $x$ \\
\hline Meetings & $x$ & $x$ & $x$ & $\checkmark$ \\
\hline Knowledgebase & $\checkmark$ & $\sqrt{ }$ & $\checkmark$ & $\checkmark$ \\
\hline $\begin{array}{c}\text { Pricing } \\
\text { (10 users per year) }\end{array}$ & $\$ 9,000 /-$ & $\$ 7,800 /-$ & $\$ 4,800 /-$ & Free \\
\hline Internal Support Tickets & $x$ & $x$ & $x$ & $x$ \\
\hline Access Control & Hierarchical & Hierarchical & Hierarchical & $x$ \\
\hline Geographical Access Control & $x$ & $x$ & $x$ & $x$ \\
\hline Geotagging (Mobile) & $x$ & $x$ & $x$ & $x$ \\
\hline Customizability & $x$ & $x$ & $x$ & $\checkmark$ \\
\hline
\end{tabular}

\subsection{Problems in Existing Systems}

The primary limitation of Salesforce CRM is the lack of an immensely customizable Campaign Management module as required by the industry. Since Salesforce expects the Small and Medium - sized Businesses to purchase a Marketing Automation software to be used for Marketing - related activities, the software is mainly fixated on managing and maintaining Sales records. Also the absence of the Campaign Analysis module in the existing Salesforce CRM system makes it impossible for the extent of a successful Campaign to be analyzed. 
Microsoft Dynamics CRM is a proprietary and comparatively more expensive software, thus reducing the probability of SMB's will buy it. To use a web resource in a visualization, Microsoft Dynamics CRM requires you to either use the SDK or import a custom visualization XML. The learning curve is complex, and as Dynamics CRM has limited plugins to choose from, you may not have some of the same functionality and performance like SuiteCRM through their marketplace of developer plugins.

SugarCRM and SuiteCRM are open source CRM software, making it simple for developers in different organizations to tailor the product in accordance to their requirements. It also allows developed versions of the CRM software to be distributed. SugarCRM, however, has a certain number of limitations, such as no support for free versions, no provisions for Campaign Analysis, and workflows that are not robust. Furthermore, SugarCRM falls short of many features which the developer demands that the users will incorporate from the modules accessible on the developer's website.

\section{PROPOSED SYSTEM}

Sales Performance Management System for Mutual Funds proposes a web application and a mobile application to better interactions between a company and its customers and

Potential customers, thereby improving profitability. The solution lets you focus on individual customer's relationships with the organization by providing the full history of all communications, meetings, and documents shared.

The implementation of this system helps businesses run smoothly by automatically updating customer data and inventory, tracking and managing sales information and upcoming events and campaigns, and generating reports to view the qualitative and quantitative performance of employees. The system will be available on mobile and desktop platforms with instant access for employees in a centralized storage.

\subsection{Event Management}

Sales Performance Management System for Mutual Funds allows differentiation between regular ongoing events and a special event, such as a promotion or a campaign, and is able to report \& analyse separately for the same, while taking it into account for the overall targets. The application enables you to plan the events, promote the events via e-mail, and track all the key components of your events entirely within the CRM system.

\subsection{Contact Management}

Contact Management provides quick access to account names and contact information, and is often used with Calendaring, E-mail Integration and Activity Management. [7] The contacts in the system refer to Distributors, Key clients, and Prospects.

The system allows us to create, change, maintain, and associate individual contacts with the Relationship Managers. The system can capture detailed distributor profile, along with other information, so users can track birthdays, anniversaries and other important dates, and send automated email greetings to them. Bulk reassigning of contacts is possible in case the Relationship Manager is getting transferred or leaving the organization.

\subsection{Qualitative Performance Tracking}

The Relationship Managers will enter the activities they performed with the contacts that have been mapped to them. They will enter their plans and schedules they have set for meeting/interacting with the Distributors/Clients. The Supervisor will be able to view the activities/plan of his team at any given point in time.

The system provides the ability to print out weekly Time Table(s), for any pre-defined period, containing a list of all the meetings/activities that a particular Relationship Manager has been involved in any or all relationships that he/she is handling.

\subsection{Opportunity Management}

A Sales Opportunity is a contact or an account which has been qualified. This person has entered into your sales cycle and is committed to working with you. You have already contacted, called, or met him/her and know their needs or requirements. [8]

A framework for representing the sales process is provided by provided by Sales Performance Management system. It allows you to track the progress of the process and adds clarity to your sales pipeline which helps you regularize your sales process and keep the opportunities advancing, get to know where you currently stand in the sales process, what the next step is and how to complete it and converge on the significant opportunities.

\subsection{Activity Management}

Activity management is an integral part of CRM, which administers all activities undertaken by the employees of the company. Any data saved in an activity is an important source of information that needs to be accessed by all relevant employees. [9]

Sales Performance Management System for Mutual Funds allows us to create daily schedulers, alerts and meeting managers. We also have the provision to reschedule meetings, over-ride pre-set meetings, skip/snooze for the week, or delegate meetings to another user. The system can capture event recurrence parameters as defined by the user. The system also allows the user to send automated e-mails and setup reminders as per the defined workflow.

\subsection{Document Repository}

The system keeps a record of all the product catalogues of several mutual fund offerings. This data can be updated on a real-time basis to reflect the quantity of resource/catalogues at hand. If a sales representative feels they are running short of such resources, they can issue a ticket to the Operations department to request to supply them with extra marketing materials, as needed. This ticket would be captured, tracked, and managed, and the respective department(s) would be notified of the same.

\subsection{Access Control}

The prevention of the use of resources in an unauthorized manner is called Access control, which is, preventing people and software who're not authorized to use those resources from doing so. Access control can be performed on a dual basis, both Hierarchical based and Geographical based. The Sales hierarchy is defined as follows, starting from the top: Sales Head, Zonal Head, Regional Head, Branch Head, Relationship Manager, and finally Distributor/Corporate, at the bottom. Users at different levels are separated hierarchically, while users at the same level are separated geographically. So, while the Branch Head can monitor the activities of the Relationship Managers under him/her, they cannot do the same for Relationship Managers under other Branch Heads. 


\subsection{Geo-Tagging}

Geo-tagging is the process of adding geographical information, such as Latitude and Longitude, to various media in the form of metadata. It is based on positions and coordinates and is often directly taken from a Global Positioning System (GPS). [11]

In this system, the location of field representatives will be recorded periodically using their device's GPS coordinates. Representatives can push their location to the back-end system every time they visit a customer/distributor for a meeting.

\subsection{Internal Support}

The system allows sales representatives across several locations to raise internal support tickets to their centralized marketing team. For example, a Branch can raise a ticket to the Head Office to send product brochures to replenish their inventory. Tickets can be tracked on a real time basis by the system to monitor the progress of the response to a request.

\subsection{Offline Mode}

The mobile application can also be used in offline mode when the Smartphone/Tablet is not connected to the Internet. In such a scenario, the user can continue using the application, performing all functions they could while connected to the Internet. Once the device gets an active Internet connection, the changes made offline can be synchronized with the centralized database by selecting "Account Sync".

\section{IMPLEMENTATION}

Sales Performance Management System for Mutual Funds will be built to be a complete package to all the problems discussed above. The system can be used to establish a hierarchy of users such as Distributor, Relationship Manager, Branch Manager, Regional Manager, Zonal Manager, etc. The system would track the performance at each level of the hierarchy. For example, it will be able to track the performance of a specific Branch by aggregating the business of all Relationship Managers under that Branch. Contacts will be stored and managed by a Primary and a Secondary Relationship Manager. Integrity and confidentiality of the system can be preserved by assigning roles and security groups to the users of the system. This system will integrate workflows to automate the processes.

Unlike most CRM software, Sales Performance Management System for Mutual Funds will be accompanied by a mobile application on Android platform to promote interaction with customers at every touch point. The mobile application will allow sales representatives to collect data and serve customers even when they are outside the office premises. Geo-tagging will be a new and unique functionality which will allow sales representatives to check-in before their meeting, so the system can verify that the meeting has occurred with the client.

\subsection{Block Diagram}

Figure 2 represents the working of Sales Performance Management System with Campaign and Sales Analysis. The system takes the Campaign activity as input and processes the Campaign Activity Response. All the targeted customers who may invest in the Mutual Funds schemes are put in the Leads table. When the Leads show interest in investing in the schemes, they are converted into Opportunities. When a transaction takes place, the Opportunities are finally classified as Customers. The system will analyse this customer data and Sales life cycle to help improve sales. The system also takes the Employee data as input and tracks it to generate a Performance Evaluation Report. Analysis of the Customer Sales Life Cycle by the system will give us the Sales Analysis. The Product data can be updated in the system on a real time basis in the form of Product Catalogue. The system uses Geotagging to track the Employees current location.

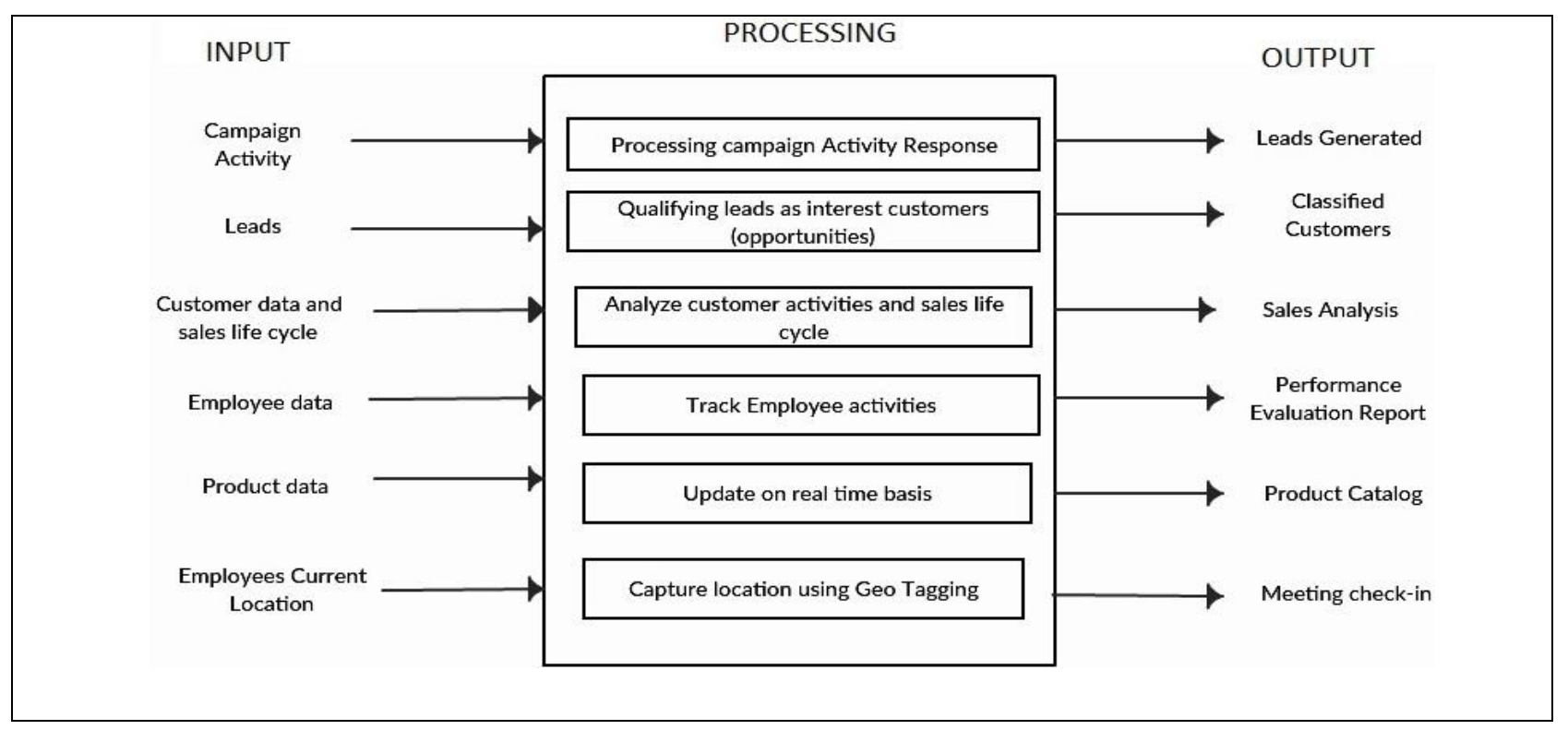

Figure 2: Block Diagram 


\subsection{Sequence Diagram}

Figure 3 shows the sequential flow of events in the system right from the point when contact management system creates new contact, Employee/User logs in, creates various tasks and to-dos on a calendar. It depicts the sequence of events in launching a Marketing Campaign and the Sales Process. Time frame describes the duration for which an activity will be active

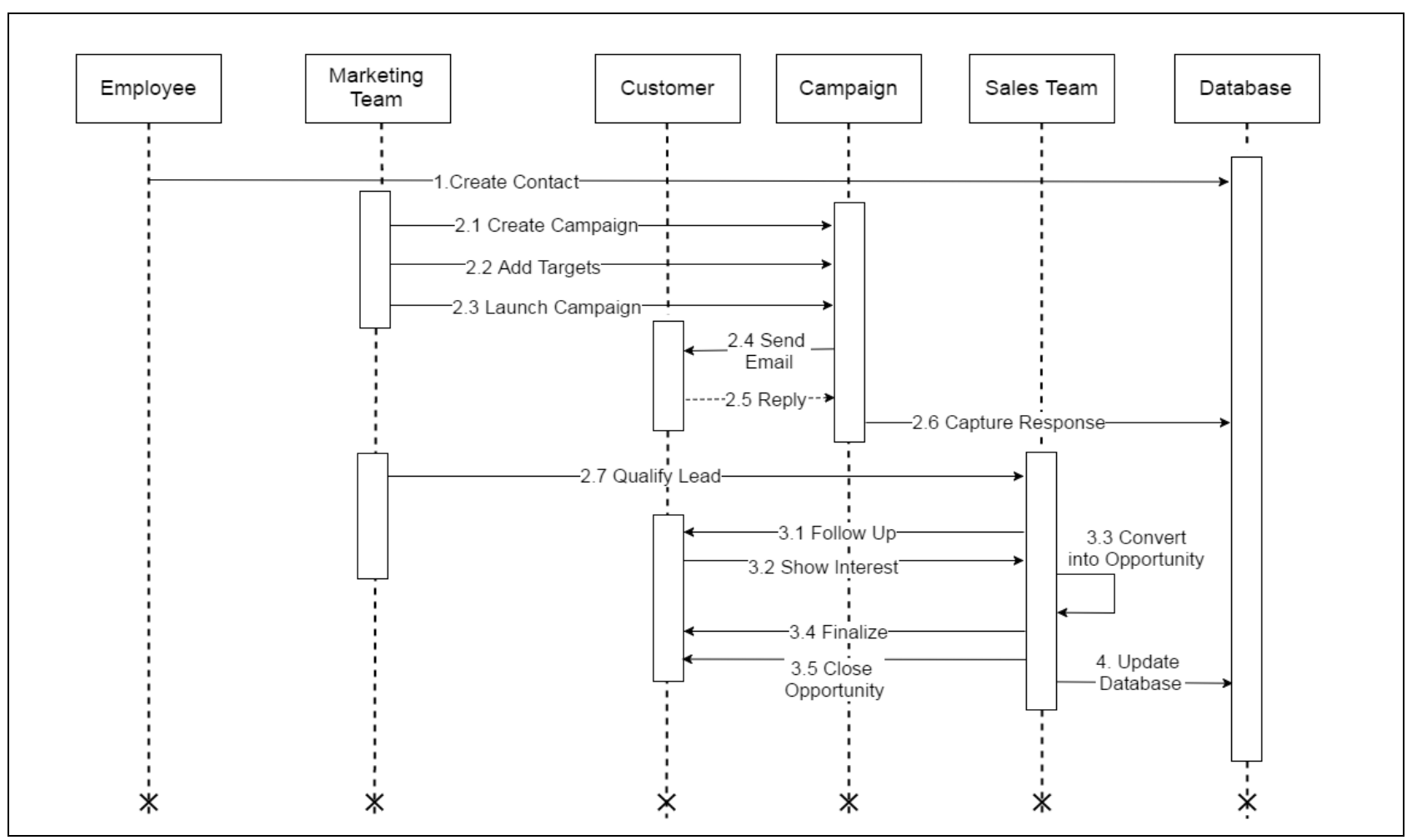

Figure 3: Sequence Diagram for Sales Lifecycle

\subsection{Flowchart}

Figure 4 represents the flowchart of the system. The user will create certain contacts and accounts, then he will create a marketing campaign. User will plan the activities related to contacts and campaign. Based on response getting from contacts using campaign, they are converted to leads. If lead is qualified then it gets converted to opportunity and if that opportunity starts business with our organization then we can close that opportunity, otherwise we stop there. If in case lead is disqualified, then we will not go for any business with that lead, we declare it as lead lost and stop there.

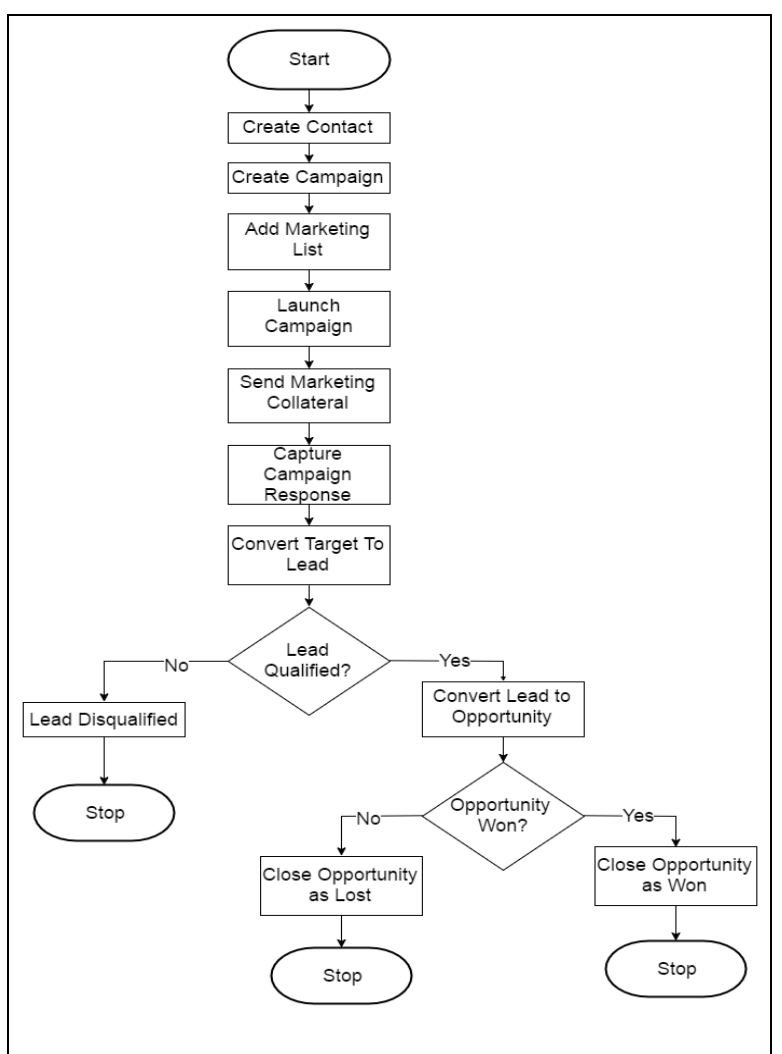

Figure 4: Flowchart of the System 


\section{TECHNOLOGIES TO BE USED 5.1 Web Application \\ 5.1.1 PHP}

This will be incorporated in the system for interacting with the MYSQL database to dynamically generate web pages. PHP script will be used to add new module, records related to each module into the database and fetch that in real time. PHP script will be used for system to automatically send out emails to individuals clients. Using PHP's dynamic pages, we aim to provide dynamic, lucid and attractive web content. PHP script will be used to code APIs (Application Programming Interface) to simplify the workflow.

\subsubsection{MySQL}

This system will use MySQL database to store records related to all modules. The main advantage is MySQL database is open source so we don't have to pay to use it. Also MySQL provides better database security by using additional location parameter that is host name or IP address while authenticating the user. This means that a user logs in to particular domain may or may not same as the same user logs in to another domain. MySQL provides hierarchical privileges.

\subsection{Mobile Application}

\subsubsection{JAVA}

The logic can be implemented using $\mathrm{C}, \mathrm{C}++$ and java but we are using java because Java is known language and large number development tools are available for it. It runs in a $\mathrm{VM}$, so no need to recompile it for every phone out there and easy to secure. Also, it easily separates processes from each other, preventing a rogue application from destroying your phone or interfering with other applications.

\subsubsection{XML}

XML stands for an eXtensible Markup Language and it is like HTML (Hyper Text Markup Language).It should be mainly used for design purposes because XML is a lightweight layout. Using XML, we can create text views, list views, etc in android which provides the users the interface to interact with the application.

\subsubsection{JSON APIs}

This system will use JSON (Javascript Object Notation) data format to transmit information in attribute-value pairs. This data is parsed in android application using the JSON APIs.

\section{SUMMARY}

Sales Performance Management System makes an effort to enhance the organization's customer base by creating targets lists, approaching them through various marketing strategies and enriched campaigning. Sales Performance Management System is an important aspect of any Mutual Funds organization that must be handled skillfully. The implementation of these processes increases efficiency, keeps the employees at the various levels of the hierarchy well informed to take important decisions. Existing systems have various missing aspects that are covered by this project, making it a better alternative.

Another highlight of this project is that it extracts knowledge from data which goes beyond the traditional charting tools to determine critical information about the effectiveness of a campaign, employee efficiency and overall organizational success.
The application also makes an effort to enhance the organization's customer base by creating targets lists, approaching them through various marketing strategies and enriched campaigning.

In addition, Sales Performance Management Systems can be transitioned from the traditional server to a cloud based architecture for business intelligence which will help to provision an up-to-date picture of customer data, sales pipelines and email. Cloud-based analytics can integrate information from multiple sources, then use data visualization or predictive modeling to support better, faster decisions. Speed to insight, as well as data sharing and collaboration is a critical benefit of cloud CRM. [12]

\section{REFERENCES}

[1]Salesforce.com "What is CRM?"https://www.salesforce.com/in/crm/what-iscrm.jsp, Date of access: 06 September, 2016

[2]TechTarget (2007). "What is CRM Analytics?"http://searchcrm.techtarget.com/definition/C RM-analytics, Date of access: 06 September, 2016

[3] PC Magazine (2016). "Salesforce Sales Cloud Lightning Professional”.http://in.pcmag.com/salesforcecomprofessional-edition/17596/review/salesforce-salescloud-lightning-professional, Date of access: 07 September, 2016

[4] Microsoft Developer Network (2011). "View data with visualizations (charts)".https://msdn.microsoft.com/enus/library/gg309647.aspx, Date of access: 07 September, 2016

[5]PC Magazine (2015). "SugarCRM Sugar Professional".http://www.pcmag.com/article2/0,2817,23 64821,00.asp, Date of access: 08 September, 2016

[6] Top Ten Reviews (2015). "The Best CRM Software of 2016".http://www.toptenreviews.com/business/software/ best-crm-software/, Date of access: 09 September, 2016

[7] CRMSearch (2008). "Differences between CRM and Contact

Management".http://www.crmsearch.com/contactmanagement-crm.php, Date of access: 10 September, 2016

[8] Pipeliner CRM Blog (2013). "What is a Sales Opportunity Management?"http://blog.pipelinersales.com/salespipeline-management/what-is-an-opportunitymanagement/, Date of access: 11 September, 2016

[9]SAP Library (2009). "Activity Management”.http://help.sap.com/saphelp_crm700_ehp0 4/helpdata/en/88/0988b891e14794a89f56bc9b00fe09/fra meset.htm, Date of access: 11 September, 2016

[10]TechTarget (2014). "What is access control?".http://searchsecurity.techtarget.com/definition/ access-control, Date of access: 12 September, 2016

[11]Techopedia (2011). "What is Geotagging?". https://www.techopedia.com/definition/86/geotagging, Date of access: 12 September, 2016

[12]Cloud Tweaks (2015) "Benefits of Cloud CRM". http://cloudtweaks.com/2015/01/business-benefits-cloudcrm/: Date of access: 7 January, 2017 